solved by science, but there are many forms of naturalism and it is unclear exactly where Ruse stands (see Susan Haack's Evidence and Enquiry (Blackwell, 1993) for clear distinctions and refutations of some forms of naturalism).

In the section on evolutionary epistemology, Ruse argues that scientific thinking (indeed, all thinking) is to be understood with the help of "epigenetic rules". These are rules of thinking that we are disposed to follow because of our evolutionary history, such as the law of non-contradiction. If our ancestors were inclined to regard sabre-toothed tigers as both dangerous and not dangerous at the same time, they would not have been our ancestors. The approach is much more promising than that of, say, the philosopher David Hull, who sees the scientific success of a theory simply in terms of the proliferation of memetic copies, without considering the rational filters at work. But, although promising, Ruse's approach shows its defects when he goes on to say that the necessity of logical rules is based on nothing but our feelings of necessity shaped by evolution. He neglects the fact that conforming to the law of noncontradiction saves us from moving in an argument from true premises to a false conclusion, and also enables us to test the implications of our theories (because a false conclusion of a valid argument implies a false premise). Logical necessity and validity are emergent properties of language and cannot be defined in terms of feelings (evolved or not).

Such a view also neglects the most interesting thing about evolution - the emergence of radically new structures and properties not fully derivable from, contained in or explicable by those that came before. Once we have language, for instance, we can build theories that no one person can fully grasp: they are no longer just a part of our psychology. Perhaps the ability to count, for example, was selected phylogenetically, but after Godel's famous incompleteness proof, we must say that the linguistically formulated theory of natural numbers has unfathomable content and that this is a fact independent of the way we feel about the proof. If this is not enough to convince Ruse that we are capable of producing systems that have independent properties, I would suggest looking at Danny Hillis's work, which suggests that self-evolving computer programs may produce perfectly working airline navigation systems containing millions of lines of program that nobody could possibly understand.

There are surely elements of marketing in the selection of scientific theories, but, if one wants to know about that, it is wise to consult advertising and marketing specialists. At the end of the day we have still to analyse what is the best method for enhancing the growth of scientific truth.
We can teach students how to falsify theories and how to obtain jobs, and with enough ingenuity, there need be no conflict.

I have focused mainly on a few problems in Evolutionary Naturalism, but it covers in an informative way many other interesting areas. In the discussion of evolutionary ethics, for example, Ruse argues that our morals are, like our logic, based on biological evolution. The book is a readable and forthright presentation of his views on the relevance of evolution to philosophy and could serve as a useful introduction for students.

Ray Percival, an associate editor of Journal of Social and Evolutionary Systems, is at 70 Hillview Court, Astley Bridge, Bolton BL1 8NU, UK.

\section{Relative values}

\section{Sean Nee}

Macroecology. By James H. Brown. University of Chicago Press: 1995. Pp. 269. \$42.50, £33.95 (hbk); \$15.95, $£ 12.75$ (pbk).

From 1736, generations of the Marsham family, who lived near Norwich, England, each year meticulously recorded facts such as the dates of the first snowdrops and cuckoos, resulting in an invaluable data set for studying how species respond to variations in climate. Darwin provides the best example of how endless catalogues of facts about natural history, each fairly dull in itself, can be illuminating when looked at, imaginatively, as a whole.

This stamp-collecting instinct is rife in ecology. Starlings weigh 80 grams and four million of them live in Sweden. Ecologists do not of course stare blankly at these facts, mesmerized. Rather, they ask how and why starling numbers have changed over time, and how a starling's weight affects its chances of surviving the winter. These questions take us in one direction, towards the particular. James Brown invites us to go in the other direction and to ask, for example, about the relationship between body size and abundance of birds in general - that is, to put all the facts into one big data set, to find empirical generalizations and to try to understand them.

Clearly, there are many permutations. If we just look at the shape of the distribution of bird body sizes, we see that it is skewed, with many more small species than medium or large ones. This is true not only for birds but for mammals, fish, trees, bacteria and insects as well, suggesting that there is a general principle to be unearthed. The main variables for which macroecologists have data are the body size, abundance, habitat specificity and geographical range of species, providing us with spaces of up to four dimensions in which to plot species points and look for interesting features in the resulting cloud. Brown demonstrates that with imaginative use of these data, meaningful hypotheses can be posed and many of their implications can be tested.

This is not a "radical new research agenda", as the publishers would have us believe, although it is interesting and important, and this is the first book to package the field for wide consumption. But it does contain some radical ideas. For example, Brown offers an argument to explain the distribution of animal body sizes that defines fitness as the rate at which animals invest energy in reproduction. Because this invites us to discard current theories of life-history evolution, the author indeed "makes a major intellectual leap", but perhaps into the abyss.

Our thinking about allometry is also challenged. It is well known that larger mammals live longer, bear fewer young, have higher metabolic rates and so on. In other words, the relationship between body size and just about everything else is mono-tonic. Brown suggests that many of these relationships may in fact be triangular, with very small-bodied species showing the opposite overall trend. If true, this would be important. But so far there is only anecdotal evidence, with one exception. Certain data sets reveal a triangular relationship between body size and abundance, with the most abundant species having intermediate sizes. But it has been argued in the literature that the relationship is merely a sampling artefact, although Brown does not mention this.

In fact, there is much that Brown does not discuss, and other macroecologists reading this book will, quite rightly, be annoyed by the extent to which their work is ignored. One is given no hint, for example, that people have been using species-area relationships to predict extinctions for decades before the publication of a paper coauthored by Brown in 1992, which gets a chapter all to itself. Nevertheless, Brown is a dominant figure in this field and his work is a suitable vantage point for an overview of the macroecological research programme.

Sean Nee is in the Department of Zoology, University of Oxford, South Parks Road, Oxford OX1 3PS, UK

The Chambers Dictionary of Science and Technology is 55 years old this year and was last revised seven years ago. The seventh edition, edited by Peter Walker, has just been published as the Larousse Dictionary of Science and Technology. Its 1,236 pages contain 49,000 entries, over 500 illustrations and many appendices. Larousse, $£ 45$ (hbk), £19.99 (pbk). 